\title{
Viral RNA Trans-Splicing: Chance Event or Product of Evolution?
}

\author{
Volker Patzel * \\ Department of Microbiology, Yong Loo Lin School of Medicine, National University of Singapore, Singapore
}

\begin{abstract}
Received: November 29, 2013; Accepted: December 02, 2013; Published: December 06, 2013
*Corresponding author: Volker Patzel, Department of Microbiology, Yong Loo Lin School of Medicine, National University of Singapore, Singapore, Tel: 65-6516-3318; Fax: 65-6777-5720; E-mail: micvp@nus.edu.sg
\end{abstract}

\section{Editorial}

One year after the discovery of exons and introns in the adenoviral hexon gene by the teams of Roberts and Sharp [1,2], Walther Gilbert suggested that the employment of different exons of a single gene could lead to the generation of various mRNA isoforms [3], a process that is today known as alternative splicing. Alternative splicing implies a number of distinct mechanisms including exon skipping, intron retention, mutually exclusive exons, alternative splice site selection as well as alternative promoter usage and alternative polyadenylation. Constitutive and alternative splicing isn't mutually exclusive which solves the former problem that for a steady evolutionary process, essential genes don't need to duplicate before one version can mutate to a new gene function. Alternative splicing generates new functions by creating new exons within existing genes and can relax the negative selection pressure against evolutionary changes in functional genes. Thus, alternative splicing might open nearly neutral paths for evolutionary changes.

For most viruses, economy in genome size is a common theme. To express a maximum number of proteins, viruses use multiple promoters, translational frame shifting, alternative open reading frames, stop codon read-through; for regulation of gene expression, viruses further use antisense transcription and virus-encoded miRNAs. Alternative splicing represents a key mechanism that is recruited by most DNA viruses, and nuclear replicating RNA viruses to generate the full repertoire of protein functions [4].

RNA trans-splicing represents a special form of alternative splicing in which sequences of distinct pre-mRNA transcripts are joined in trans. Thus, like alternative cis-splicing, trans-splicing contributes to the diversification of genotypes and phenotypes. Naturally occurring RNA trans-splicing was first described in 1986 in trypanosomes and one year later also in the nematode Caenorhabditis elegans. In these organisms a species-specific non-coding small RNA with a singular 5' splice donor site, the socalled small leader RNA (SL RNA), is spliced to variable 3' splice acceptor sites on separate pre-mRNA molecules. SL RNA transsplicing differs from regular cis-splicing, as the U1 small nuclear ribonucleoprotein (snRNP) containing the U1 small nuclear (sn)
RNA is replaced by the SL snRNP, which carries the SL RNA. SL RNA trans-splicing was also detected in other nematodes, flatworms, and in tunicates, primitive chordates. The hypothesis it might also occur in vertebrates has not been confirmed yet. In the mid-90s first experimental evidence was obtained showing that mammalian cells can combine natural pre-mRNAs to new mRNA via RNA trans-splicing $[5,6]$. The mammalian RNA transsplicing, however, is independent of SL RNA but instead mediated by the spliceosome of the cell. Computational analyses of cDNAs from a gene databank indicated one percent of all sequenced mRNAs to be chimeric [7], some of which might be synthesized by RNA trans-splicing. Up to date several examples of naturally occurring mammalian RNA trans-splicing have been reported [814].

A major difference between cis- and trans-splicing relies in the fact that the efficiency of trans-splicing strongly depends on the probability that two separated RNA precursor molecules encounter each other in the nuclear compartment. This likelihood is maximal, when the two RNA precursors originate from the same transcriptional locus and when they contain antisense binding domains to trigger kind of intron-like bridging between splice sites. Splicing between two identical RNA transcripts is called 'homologous' trans-splicing which typically results in larger mRNAs with complete or partial exon duplications. Homologous trans-splicing was firstly demonstrated for viral RNAs [5] and later also for mammalian primary transcripts [8]. Trans-splicing between different RNAs originating from different transcriptional loci is termed heterologous trans-splicing. The pre-mRNAs undergoing heterologous trans-splicing can originate from different species, e.g. a virus and the infected host cell, or the same species. Though being kinetically less favoured examples of cross-species or species internal heterologous transsplicing were reported. These include trans-splice combinations between two viruses as SV40 and HIV [9], or between cellular RNA sequences and viral RNAs like HIV-nef pre-mRNA [9], the adenoviral major late transcript [11], or a HCV transgenic transcript [14]. The examples reported so far impressively demonstrate a frequent involvement of viral transcripts in transsplicing reactions. A common theme of all naturally occurring trans-splicing events involving viral RNA transcripts is the 
employment of cryptic splice sites and/or stand-alone regular cis splice sites that lost their cis splice-site counterparts by mutation. Mutational splice site weakening is not unlikely to occur during erroneous viral replication.

For the reasons discussed above it is reasonable to assume that in particular viruses, as the smallest replicating specimen, use the mechanism of RNA trans-splicing to increase the codon complexity of their genomes at the pre-mRNA processing stage in order to maximise their transcriptomes and proteomes. Such a nearly neutral evolutionary path is certainly less relevant in acute viral infections where large numbers of viral particles are produced with high error rates of replication. However, that might be different in the course of persistent viral infections which require a more precise control of viral replication.

Our recent finding that the SV40 virus uses homologous RNA trans-splicing to generate a functional protein, namely a highly transforming $100 \mathrm{kD}$ super $\mathrm{T}$ antigen, supports these hypotheses [15]. Homologous SV40 RNA trans-splicing occurred between the early viral transcripts and was enhanced in clones carrying a single point mutation in the large $\mathrm{T}$ antigen $5^{\prime}$ donor splice site (ss). This mutation impaired cis-splicing in favour of an alternative trans-splice reaction via a cryptic 5'ss within a second cis-spliced SV40 pre-mRNA molecule and enabled detectable gene expression. Intriguingly, SV40 RNA trans-splicing seems to be supported by a set of molecular trans-splice helper functions including mutation-based weakening of the regular 5' cis ss, the absence of an alternative $5^{\prime}$ cis ss upstream and the presence of an alternative cryptic 5'ss downstream of the 3'ss, whereby the use of that cryptic 5'ss is supported by splice enhancer sequences and two pairs of RNA dimerization domains. The concerted occurence of these supporting genetic functions likely is not coincidental but instead these functions constitute intrinsic characteristics of the viral sequences leading them to transsplicing. As a consequence, homologous SV40 RNA trans-splicing was found to be a highly efficent process with $50 \%$ or more viral transcripts being involved in the trans-splice reaction.

In conclusion, SV40 RNA trans-splicing is certainly not a chance event but instead a mechanism that is intrinsic to the virus to increase protein variation. It is reasonable to assume that other viruses use the mechanism of trans-splicing to generate new gene products as well. As reported for the SV40 virus, alternative RNA cis-splicing of long pre-mRNA transcripts originating from genomic viral tandem integration can trigger the generation of the same transcripts as RNA trans-splicing. However, RNA transsplicing is independent of genomic integration and, thus, could be favoured during episomal viral infections. One could further speculate that besides by DNA recombination, novel viral RNA genomes, i.e. those of retroviruses, recombine in the nucleus of infected cells through the mechanism of RNA trans-splicing.

\section{Acknowledgment}

This work was supported by the NUS-Cambridge Start-up grant of the National University of Singapore [grant number R-182-000-163-646]; the National Medical Research Council of Singapore [grant number NMRC/NIG/1058/2011] and the Academic Research Fund of the Ministry of Education of Singapore [grant number R-182-000-200-112].

\section{References}

1. Berget SM, Sharp PA (1977) A spliced sequence at the $5^{\prime}$-terminus of adenovirus late mRNA. Brookhaven symposia in biology, 29: 332-344.

2. Chow LT, Gelinas RE, Broker TR, Roberts RJ (1977) An amazing sequence arrangement at the $5^{\prime}$ ends of adenovirus 2 messenger RNA. Cell, 12:1-8.

3. Gilbert W. Why genes in pieces? (1978) Nature, 271: 501.

4. Hernandez Lopez HR, Graham SV (2012) Alternative splicing in human tumour viruses: a therapeutic target? Biochem. J, 445(2): 145156.

5. Eul J, Graessmann M, Graessmann A (1995) Experimental evidence for RNA trans-splicing in mammalian cells. EMBO J, 14(13): 3226-3235.

6. Eul J, Graessmann M, Graessmann A ( 1996) In vitro synthesized SV40 cRNA is trans-spliced after microinjection into the nuclei of mammalian cells. FEBS Lett, 394(2): 227-232.

7. Romani A, Guerra E, Trerotola M, Alberti S. Detection and analysis of spliced chimeric mRNAs in sequence databanks. Nucleic Acids Res, 31(4): e17.

8. Caudevilla C, Serra D, Miliar A, Codony S, Asins G, et al. (1998) Natural trans-splicing in carnitin octanoyltransferase pre-mRNAs in rat liver. Proc Natl Acad Sci USA, 95(21): 12185-12190.

9. Caudevilla C, Codony C, Serra D, Plasenica G, Roman R, et al. (2001) Localization of an exonic splicing enhancer responsible for mammalian natural trans-splicing. Nucleic Acids Res, 29(14): 3108-15.

10. Flouriot G, Brand H, Seraphin B, Gannon F (2002) Natural transspliced mRNAs are generated from the human estrogen receptoralpha (hER alpha) gene. J Biol Chem, 277(29): 26244-26251.

11. Kikumori T, Cote GJ, Gagel RF (2002) Naturally occurring heterologous trans-splicing of adenovirus RNA with host cellular transcripts during infection. FEBS Lett, 522(1-3): 41-46.

12. Jehann Z, Vallinayagam S, Tiwari S, Pradhan S, Singh L, et al.(2007) Novel noncoding RNA from human Y distal heterochromatic block (Yq12) generates testis-specific chimeric CDC2L2. Genome Res, 17(4): 423-40.

13. Brooks YS, Wang G, Yang Z, Smith KK, Bieberich E, et al. (2009) Functional pre- mRNA trans-splicing of coactivator CoAA and corepressor RBM4 during stem/progenitor cell differentiation. J Biol Chem, 284(27): 18033-18046.

14. Desai MM, Tumurbataar S, Zhang Y, Chan LN, Sun J, et al. (2011) Aberrant transcription and post-transcriptional processing of hepatitis $\mathrm{C}$ virus non-structural genes in transgenic mice. Transgenic Res, 20(6):1273-1284.

15. Eul J, Patzel V (2013) Homologous SV40 RNA trans-splicing: A new mechanism for diversification of viral sequences and phenotypes. RNA Biology 10, (11):1-11. 